\title{
Billiards with Pesin Region of Measure One
}

\author{
Roberto Markarian \\ Instituto de Matemática y Estadística "Prof. Ing. Rafael Laguardia", \\ Facultad de Ingeniería. Universidad de la República, Montevideo, Uruguay
}

\begin{abstract}
We give a description of a large class of plane billiards with Pesin region of measure one. Open conditions including properly those founded by Wojtkowski [W1] for $C^{4}$ focusing boundaries are obtained. Lyapunov's forms, introduced by Lewowicz, are used.
\end{abstract}

\section{Introduction}

Boltzmann's hypothesis about the ergodicity of the model (Boltzmann-Gibbs) of the gas of hard spheres is in the basis of the study of the statistical properties of dynamical systems. Sinai's proof [S1] of the simplest case of this hypothesis concerns the plane with two balls and a square box represented by the torus $\{(x, y) \bmod 1\}$. This situation is immediately extended for billiards with boundaries of negative curvature (dispersing billiards).

Birkhoff, Krylov, and Arnold gave intuitive interpretations of the relations between the dynamical systems of the billiard type and the geodesic flows of surfaces. The study of ergodic properties of these flows in surfaces of negative curvature showed the importance of the transversal stable and unstable foliations (separately, Hopf and Hedlund, 1939). This methods were profitably used since the sixties in the study of the so-called Anosov systems and other topics [see for example (A1)].

The above mentioned relations justify the use of the same techniques in the study of ergodic properties of billiards with dispersing boundaries. This is the central idea od Sinai's work.

In the billiards with focusing boundaries the situation is more complicated and the pioneer works of Birkhoff suggested the non-ergodicity of plane billiards with boundaries that consist of a smooth curve of positive curvature. Bunimovich [B2, B 3], indicated that billiards whose focusing pieces of the boundary have constant curvature and that do not contain dispersing components, are Bernouilli (the stadium, for example). 
In [W1] it was proven that some examples of billiards (the cardioid, an epycycloid, a square with a hole of the shape of an astroid) have Pesin region of measure one. Besides, this property is preserved under small perturbations of their boundaries. In this work it is proved that the condition obtained by Wojtkowski for focusing curves is not generic. More precisely, open conditions for focusing $C^{4}$ curves, different than those in [W1], are obtained.

We use procedures [L1-L4] which permit the study of all this properties looking at the asymptotic behaviour of certain quadratic forms. The existence of contracting and expanding subspaces detected by these forms (see the Appendix) permit the application of Pesin's theory (see [P1, pp. 58, 61-62, 71-73] which guarantee the construction of local stable and unstable foliations that generally are not uniform, neither in length nor in their angle, but are sufficient to obtain certain ergodic properties.

\section{Notation and Wave Fronts}

Following Cornfeld-Sinai-Fomin $[\mathrm{C} 1]$, with $Q_{0}=\mathbb{R}^{2}$, let

$$
M_{1}=\{x \in \partial \tilde{M}:(n(q), x)>0, q=\pi(x)\},
$$

where $n(q)$ is the unit inward normal vector at

$$
q \in \partial \widetilde{Q}_{i}=\partial Q_{i} \backslash \bigcup_{k \neq i} \partial Q_{k},
$$

$\partial \widetilde{Q}_{i}$ being a regular component of the boundary; $\pi$ is the natural projection from the tangent bundle to $\mathbb{R}^{2}, \pi(q, v)=q ; \partial \tilde{M}_{i}=\pi^{-1}\left(\partial \widetilde{Q}_{i}\right) ; \partial \tilde{M}=\bigcup \partial \tilde{M}_{i}$.

If $s$ is the arc length of the parametrization $\alpha(s)$ of the component $\partial Q_{i}$ of the boundary, the curvature $k(s)$ is defined by $\alpha^{\prime \prime}=\frac{d \mathbf{t}}{d s}=k n=k i \alpha^{\prime}$. Then we have regular components of the boundary $\partial Q^{+}, \partial Q^{-}, \partial Q^{0}$, respectively with positive (focusing components), negative (dispersing components) and zero curvature (neutral components).

Given $x=(q, v) \in M_{1}, T x$ (if it exists) is obtained moving forward, in the billiard surface, in the direction $v$, a distance (time) $f(x)$ till the intersection with $\partial \widetilde{Q}_{j}$ in $q_{1}$. $T x=\left(q_{1}, w\right)$, where $w=v-2\left(n\left(q_{1}\right), v\right) n\left(q_{1}\right)$ : the angle of incidence equals the angle of reflection. (Differences with the notations of the first publications of Sinai, Bunimovich, Gallavotti, etc. should be observed.)

Fig. 1

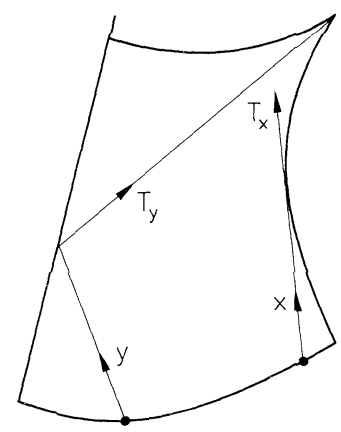


The billiard transformation $T: H \rightarrow H$ is measurable, bijective, continuous and $v$-measure preserving. Here $H=M_{1} \backslash K$, where $K$ contains the points of $M_{1}$ such that $T^{k}$ is not defined or not continuous (see Fig. 1, where $x, y \in K$ ); furthermore has $v$-measure zero. As usual $d v=d s d \theta \cos \theta$, where $\theta$ is the angle between $n(q)$ and $v ; v$ is normalized. $T: H \rightarrow H$ is a diffeomorphism of order $p-1, p$ being the differentiability order of $\partial Q$.

A wave front is given by a curve $(\alpha(s), v(s))$ in $M_{1}$, where $v(s)$ is the vector of the front, and its state after colliding with $\partial \widetilde{Q}_{j}$ is $(\gamma(\sigma(s)), w(\sigma(s)))$ (see Fig. 2). So, we can write $T(\alpha, v)=(\gamma, w)$. The symbol ' will designate the derivate with respect to $s$ or $\sigma$. Then

$$
\begin{aligned}
v(s) & =i \alpha^{\prime}(s) e^{i \theta(s)}, \\
w(s) & =i \gamma^{\prime}(\sigma(s)) e^{i \delta(\sigma(s))}, \\
\gamma(\sigma(s)) & =\alpha(s)+f(s) i \alpha^{\prime}(s) e^{i \theta(s)} .
\end{aligned}
$$

It is simple to prove the following formulas:

$$
\begin{gathered}
k_{0}+\frac{d \theta}{d s}=\left(k_{1}-\frac{d \delta}{d \sigma}\right) \frac{d \sigma}{d s}, \\
\cos \theta=\frac{d \sigma}{d s}\left[f\left(k_{1}-\frac{d \delta}{d \sigma}\right)-\cos \delta\right],
\end{gathered}
$$

where $k_{0}, k_{1}$ are respectively, the curvatures in $\alpha\left(s_{0}\right), \gamma\left(\sigma_{0}\right)$. These expressions correspond to Lemma 2.3 of Sinai [S1], adjusted in Lemma 3 of [B2], and are important for constructing the locally contracting and expanding fibres.

Fig. 2

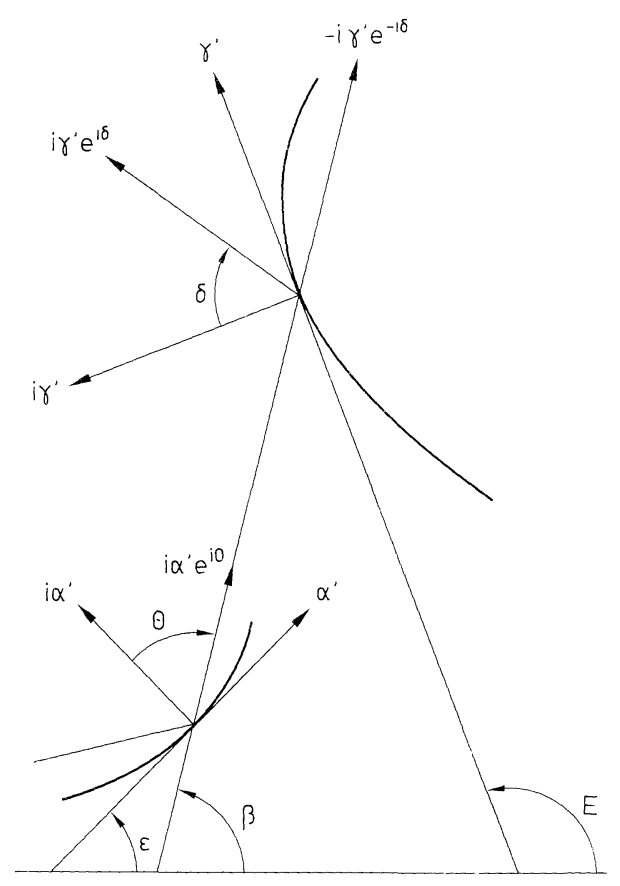


If $M_{1}$ is parametrized by $(s, \beta)$ instead of $(s, \theta)$, where $\beta$ is the angle formed by a fixed axis with $v$, we have

$$
k_{0}+\theta^{\prime}=\frac{d \beta}{d s}, \quad-\delta^{\prime}+k_{1}=\frac{d \beta}{d \sigma} .
$$

\section{Quadratic Forms}

Given a manifold $M, B: T M \rightarrow R$ is a quadratic form in $M$ if $B_{x}=B_{T_{x} M}$ is a quadratic form in the usual sense, $B$ is non-degenerate if for every $x, B_{x}$ is not degenerate. $B$ is positive if $B_{x} v>0$ for every $x \in M, v \in T_{x} M, v \neq 0$. If $f$ is a diffeomorphism and $B$ a quadratic form in $M$, we denote by $f^{\#} B$ (pull-back of $B$ by $f)$ the quadratic form defined by $\left(f^{\#} B\right)_{x} u=B_{f(x)}\left(f_{x}^{\prime}(u)\right)$.

This work continues the application of quadratic forms to investigate stability and ergodic properties of dynamical systems, begun by Lewowicz in [L1] (cf. [L 2-4]).

A proof of a theorem similar to the following one is suggested in [L 3], and given in details, in the appendix.

$\Sigma(f)$ will denote the Pesin region of $f$, that is, the set of regular points that have non-zero Lyapunov exponents. Notations are as in Mañé [M1].

Theorem. Let $f: H \rightarrow H$ be a $C^{r}$-diffeomorphism, $r \geqq 1$, that preserves the smooth measure $v ; H=H_{1} \backslash K . H_{1}$ is a compact 2-manifold,

$$
v(K)=0 ; \log ^{+}\left\|\left(f^{ \pm 1}\right)_{x}^{\prime}\right\| \in L^{1}(H, v)\left(\log ^{+} s=\max \{0, \log s\}\right) .
$$

Let $B: T H_{1} \rightarrow R$ be a bounded quadratic form, continuous and not degenerate in $T H$, such that $P_{x}=\left(f^{\#} B-B\right)_{x}$ is positive for every $x \in H$, and

$$
\begin{gathered}
S_{x}=\left\{u \in T_{x} H: B\left(\left(f^{n}\right)^{\prime} u\right)<0, n \geqq 0\right\} \\
U_{x}=\left\{u \in T_{x} H: B\left(\left(f^{n}\right)^{\prime} u\right)>0, n \leqq 0\right\} .
\end{gathered}
$$

Then $v(\Sigma(f))=1$ and the proper spaces of the negative (positive) Lyapunov exponent in $x$ is $S_{x}$ (respectively $U_{x}$ ).

The theorem is valid if $P$ is positive eventually: for every $n \in \mathbb{N}$ there exists $k \in \mathbb{N}$ such that $\left.B\left(\left(f^{k+1}\right)^{\prime}\right) u\right)-B\left(\left(f^{k}\right)^{\prime} u\right)>0$ for every $u \in T_{x} H, u \neq 0$ and almost every $x \in H$.

The proof that the billiard transformation $T$ verifies the conditions of the theorem are given, in details, in Part V (Plane Billiards as Smooth Dynamical Systems, by M. Strelcyn) of [K1]. In particular, to apply Corollary 4.1 and Theorem 5.1, it is needed for $\partial \widetilde{Q}_{i}$ to be $C^{2}$ and for $|k|$ to be uniformly bounded.

In order to define a quadratic form on $T H$ in the case of billiards we must consider curves $x(t)=(\alpha(s(t)), v(s(t)))$ in $H$ such that $x(0)=x$ and assign to each vector $u=\left.(\overline{\alpha, v})\right|_{t=0}$ a real number $B_{x} u$. 'indicates derivative with respect to $t$.

Here we study, three different forms, whose expressions are based in methods employed to study stability problems of geodesic flows [L4].

A. $B_{x} u=\left(\dot{\alpha}_{0}, i v_{0}\right)\left(\dot{v}, i v_{0}\right)=-\dot{s}_{0}^{2} \cos \theta_{0}\left(k_{0}+\theta_{0}^{\prime}\right)$. 
Using (3) we obtain $B_{x} u=-\cos \theta_{0} \frac{d s}{d t} \frac{d \beta}{d t}$, where $\left\{\frac{d s}{d t}, \frac{d \beta}{d t}\right\}$ is a basis of the tangent space $T_{x} H$. Then $B_{x}$ is a quadratic form with proper values $\pm a / 2$, $a=\cos \theta_{0}$.

$$
P_{x} u=\left(T^{\#} B-B\right)_{x} u=\left(\dot{s} \sigma^{\prime}\right)^{2}\left[-2 k_{1} \cos \delta+f\left(k_{1}-\delta^{\prime}\right)^{2}\right],
$$

which is definite positive (eventually) if the regular components of the boundary are dispersing or neutral and the trajectories pass by the not neutral components in finite time [i.e., for almost every $x$, there exist $k \in \mathbb{N}$ such that $\pi\left(T^{k} x\right)$ is in a not neutral component]. This remark on trajectories eventually going through the not neutral pieces is valid in each of the following cases.

Sinai [S1] proved that these billiards are ergodic (actually they are Bernouilli). So our result on the full measure of the Pesin region is weaker.

$$
\begin{aligned}
& \text { B. } B_{x} u=f(\dot{v}, i v)^{2}+2(\dot{\alpha}, i v)(\dot{v}, i v)=\dot{s}^{2}\left(k_{0}+\theta^{\prime}\right)\left[f\left(k_{0}+\theta^{\prime}\right)-2 \cos \theta\right. \\
&=f\left(\frac{d \beta}{d t}\right)^{2}-2 \cos \theta \frac{d s}{d t} \frac{d \beta}{d t} ; \text { with proper values }\left(f \pm \sqrt{f^{2}+a^{2}}\right) / 2 \\
& P_{x} u=\left(\dot{s} \sigma^{\prime}\right)^{2}\left[\left(f_{1}+f\right) \delta^{\prime 2}-2 k_{1}\left(f-f_{1}\right) \delta^{\prime}+k_{1}\left(f_{1}+f\right)-4 k_{1} \cos \delta\right]
\end{aligned}
$$

where $f_{1}$ is the distance between $T x$ and $T^{2} x$.

If $k_{1} \leqq 0, P$ is definite positive (eventually).

If $k_{1}>0, P$ is definite positive if the discriminant of the expression in brackets as a polynomial in $\delta^{\prime}$ is negative: $-4 k_{1}^{2} f f_{1}+4 k_{1}\left(f_{1}+f\right) \cos \delta<0$. If $L_{1}$ is the time that the trajectory spends inside the osculating circle of radius $R_{1}=1 / k_{1}$ before (or after) colliding with the boundary at $\gamma\left(\sigma_{0}\right)$, we have $\cos \delta=\frac{L_{1} k_{1}}{2}$ (see Fig. 3), and $P_{x}>0$ when the trajectory collides a focusing component of the boundary if and only if

$$
L_{1}\left(f_{1}+f\right)<2 f f_{1} .
$$

C. $B_{x} u=L(\dot{v}, i v)^{2}+2(\dot{\alpha}, i v)(\dot{v}, i v)$

if $k \leqq C, L=0$

if $k>0, L$ is the time that the trajectory spends in the osculating circle of $\alpha(s)$ before (or after) colliding.

Fig. 3

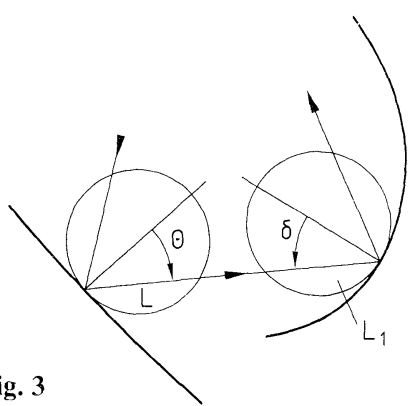


In this case

$$
P_{x} u=\left(\dot{s} \sigma^{\prime}\right)^{2}\left[\delta^{\prime 2}\left(L_{1}-L+2 f\right)+2 k_{1} \delta^{\prime}\left(L_{1}+L-2 f\right)+k_{1}^{2}\left(L_{1}-L+2 f\right)-4 k_{1} \cos \delta\right] \text {. }
$$

So, when $k_{1} \leqq 0, k_{0}>0, P_{x}>0$ if the dispersing or neutral components do not intersect the semicurvature circles of $\partial Q_{i}(\alpha(s)): 2 f-L>0$. When $k_{0}, k_{1} \leqq 0$ there is no additional condition. If $k>0$, the semicurvature circle is, by definition, inside the billiard, tangent to the boundary and has radius equal to $R / 2$. When $k_{1}>0$, a similar calculus gives a necessary and sufficient condition:

$$
L+L_{1}<2 f \text {. }
$$

\section{Conditions on the Boundaries. New Results}

We study here, separately, Cases B and C of the previous section.

$B$. In the case of the form $B_{x} u=f(\dot{v}, i v)^{2}+2(\dot{\alpha}, i v)(\dot{v}, i v)$ the conditions of the theorem are verified if $\pi(T x)$ is in a neutral or dispersing component of the boundary. If $\pi(T x)$ is in a focusing component (4) gives a necessary and sufficient condition. In this case, if

$$
L_{1}<f, f_{1}
$$

condition (4) is fulfilled and the hypotheses of the theorem are verified if the osculating circles in points of any focusing component do not intersect any other component of the boundary. So the interior angles between two focusing components must be bigger than $\pi$, and the interior angles between a focusing component and a not focusing component must be not less than $\pi$ (in case the not focusing component is neutral the angle can be bigger than $\pi / 2$ ).

But (6) is not fulfilled in general if two successive collisions are in the same focusing component. Let $\gamma(A)=r(A) \gamma^{\prime}(0) e^{i A}, r(0)=0$, the boundary curve in a neighbourhood of the collision point $\gamma(0)$ (Fig. 4).

$$
\begin{gathered}
f_{1}=r(A)=\dot{r}(0) A+\ddot{r}(0) A^{2} / 2+\dddot{r}(0) A^{3} / 6+\ldots, \\
f=-r(-A)=\dot{r}(0) A-\ddot{r}(0) A^{2} / 2+\dddot{r}(0) A^{3} / 6+\ldots, \\
A-\delta=\pi / 2, L_{1}=2 R \sin A=\frac{2}{k(0)}\left(A-A^{3} / 6+\ldots\right) .
\end{gathered}
$$

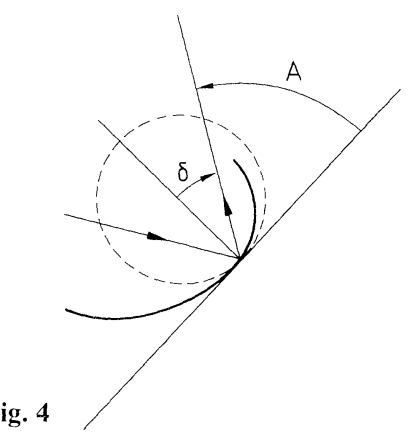


Condition (4) is written $\frac{2}{k_{1}} \sin A(r(A)-r(-A))<-2 r(A) r(-A)$, and if the curve is symmetric, $r(A)=-r(-A)$, this simple condition is obtained

$$
\sin A<r(A) \frac{k(0)}{2} \text {. }
$$

In the general case, using Taylor's development, for small angles $A$, we obtain

$$
\frac{\ddot{r}^{2}(0)}{4}<\frac{\dot{r}(0)}{3 k_{1}}+\frac{\dddot{r}(0)}{3 k_{1}} \Leftrightarrow 3 k \ddot{k}<2 \dot{k}^{2} .
$$

Or, using derivatives with respect to $s$,

$$
3 k k^{\prime \prime}<4 k^{\prime 2} \Leftrightarrow \frac{d^{2}\left(R^{1 / 3}\right)}{d s^{2}}>0 .
$$

Observe that (7) is a condition valid in a point, for trajectories coming from any point of the same focusing component of the boundary. But as (4) is an open condition, (7) holds, for long trajectories, in some neighbourhood of the point [cf. (2)].

Observe also that (8) is an open condition valid for $C^{4}$-curves, but it guarantees only the positivity of $P_{x}$ for trajectories close to tangent lines which is the situation more difficult to elucidate. So, curves that verify simultaneously conditions (7) and (8) give open $C^{4}$ families that satisfy (4) for consecutive collisions in the same focusing component.

If $r(A)=A,-A_{0}<A<A_{0}$, (7) and (8) are verified.

In the case of the ellipse of Fig. 5 ,

$$
r(A)=\frac{2 a^{2} b \operatorname{tg} A}{\left(b^{2}+a^{2} \operatorname{tg}^{2} A\right) \cos A}, \quad-\pi / 2<A<\pi / 2, k(0)=\frac{b}{a^{2}},
$$

and condition (7) is equivalent to $a^{2}<b^{2}$; so $A=0$ correspond to a vertex of the longest axis. If the ellipse is parametrized in the usual way: $x=\cos t, y=b \sin t$, $-\pi / 2 \leqq t<3 \pi / 2,(8)$ is verified, for $b^{2}>a^{2}$ if $\sin ^{2} t>\frac{b^{2}}{b^{2}+a^{2}}$, which corresponds, in
the first quadrant, to the part emphasized.

$C$. If $B_{x} u=L(\dot{v}, i v)^{2}+2(\dot{\alpha}, i v)(\dot{v}, i v)$, conditions for the validity of the theorem have been given in the cases of dispersing components, condition (5) is verified if the semicurvature circles do not intersect themselves.

Fig. 5

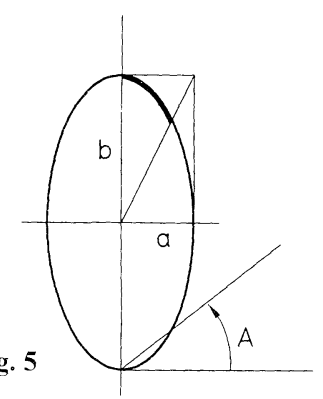


If two consecutive collisions are in the same focusing component of the boundary, (5) is equivalent [W 1] to

$$
2 k^{\prime 2}<k k^{\prime \prime} \Leftrightarrow R^{\prime \prime}(s)=\frac{d^{2} R}{d s^{2}}<0 .
$$

Condition (9) has the advantage of being global and is verified by arcs of the epicycloid, hypocycloid, cycloid. In particular, by the cardioid $r(A)=1+\cos A$. In the case of the ellipse $x=a \cos t, y=b \sin t,-\pi / 2 \leqq t<3 \pi / 2$, if $b^{2}>a^{2}$, (9) is verified if $\cos 2 t>0 \Rightarrow-\pi / 4<t<\pi / 4$ or $3 \pi / 4<t<5 \pi / 4$. This is a part of the ellipse disjoint of the one indicated in the preceding section.

\section{Conclusion}

In this work we prove the existence of two types of billiards with Pesin region of measure one, with open focusing conditions in the curvatures. This allows us to make small $C^{4}$ modifications in the boundaries, and maintain ergodic properties.

The $C^{2}$-components of the boundary can be of any type, with the following sufficient conditions (which are not necessary in general).

a) In Case B of this section, the focusing components verify (4) - with the observations of expressions (7) and (8) for permitted $C^{4}$ curves -; the osculating circles of adjacent focusing components do not intersect not adjacent components of the boundary; adjacent focusing components form interior angles bigger than $\pi$; focusing and dispersing components form interior angles not less than $\pi$; focusing and neutral components have interior angles bigger than $\pi / 2$.

b) In Case $\mathrm{C}, \mathrm{C}^{4}$-focusing components verify (9), semicurvature circles of focusing components are disjoint; components with $k \leqq 0$ do not intersect the semicurvature circles of focusing components; the conditions for adjacent components are as in a).

Billiards of type b) are those studied by Wojtkowski. But the class of billiards of type a) is large enough to prove that his assertion [W 1, p. 412] that "typically" condition (9) "is in some sense a necessary condition for nonvanishing of Lyapunov exponents" is not true.

\section{Appendix}

Proofs of the following lemmas are suggested in [L3].

Lemma 1. Let $f: H \rightarrow H$, be a $C^{r}$-diffeomorphism, $r \geqq 1 ; v$ an $f$ invariant measure; $H=H_{1} \backslash K ; H_{1}$, a compact 2-manifold; $v(K)=0$. Let $B: T H_{1} \rightarrow R$, be a quadratic form bounded on $T H_{1}$, continuous and not degenerate on $T H$ such that $P_{x}=\left(f^{\#} B-B\right)_{x}$ is definite positive $v$-a.e. $x \in H$.

Then $v$-a.e. $x \in H$,

$$
\begin{aligned}
& S_{x}=\left\{u \in T_{x} H: B\left(\left(f^{n}\right)^{\prime} u\right)<0, n \geqq 0\right\}, \\
& U_{x}=\left\{u \in T_{x} H: B\left(\left(f^{n}\right)^{\prime} u\right)>0, n \leqq 0\right\}
\end{aligned}
$$


are one dimensional linear subspaces of $T_{x} H$ that depend continuously on $x$, and $T_{x} H=S_{x} \oplus U_{x}$.

Proof. For $n>0$ let $w_{n} \in T_{f^{n}(x)} H$ be such that $B_{f^{n}(x)} w_{n}<0$.

Since $P_{x}>0, B_{x}\left(\left(f^{-n}\right)^{\prime} w_{n}\right)<0$. But $\left\{\left(f^{-n}\right) w_{n}\right\}$ has a convergent subsequence, say, to $w_{\infty}$ with $\left(f^{n_{i}}\right)^{\prime} w_{n_{t}}$ convergent to $\left(f^{n_{i}}\right)^{\prime} w_{\infty}$, and so if $B\left(\left(f^{N}\right)^{\prime} w_{\infty}\right) \geqq 0$ for some $N \geqq 0, B\left(\left(f^{n_{j}}\right)^{\prime} w_{\infty}\right)>0$ for $n_{j}>N$ and the same inequality is valid in some neighbourhood of $\left(f^{n}\right)^{\prime} w_{\infty}$. Then $B\left(\left(f^{n}\right)^{\prime} w_{\infty}\right)<0 \forall n \geqq 0, w_{\infty} \in S_{x}$ and $S_{x}$ contains a one dimensional subspace. Idem for $U_{x}$.

Let now $u, v \in S_{x}$ be linearly independent vectors and $D$ compact, $D \subset H$, such that $f^{n_{k}}(x) \in D$ for an increasing sequence $\left\{n_{k}\right\}$. The existence of $D$ is a consequence of the Poincaré recurrence theorem.

Since $P>0$ and $D$ compact there exist $b, c>0$ such that $c\|w\|^{2} \leqq P_{y} w \leqq b\|w\|^{2}$, for every $\quad w=\left(f^{n_{k}}\right)^{\prime} u, \quad u \in T_{y} H, \quad y=f^{n_{k}}(x), \quad k \in \mathbb{N}$. Then $B\left(\left(f^{m}\right)^{\prime} u\right)-B(u)$ $=\sum_{i=0}^{m-1} P\left(\left(f^{i}\right)^{\prime} u\right) \geqq c \sum_{k=1}^{n_{N}}\left\|\left(f^{n_{k}}\right)^{\prime} u\right\|^{2}, n_{N} \leqq m-1 \leqq n_{N+1}$ and if $\left\|\left(f^{n_{k}}\right)^{\prime} u\right\| \rightarrow 0$ we have $B\left(\left(f^{m}\right)^{\prime} u\right) \rightarrow+\infty$ for $m \rightarrow+\infty$, which is a contradiction. Then $\lim _{k \rightarrow+\infty}\left\|\left(f^{n_{k}}\right)^{\prime} u\right\|=0$ and

$$
\lim _{k \rightarrow+\infty}\left\|\left(f^{n_{k}}\right)^{\prime}(\lambda u+\mu v)\right\|=0 \quad \lambda, \mu \in R .
$$

This implies $B\left(\left(f^{n}\right)^{\prime}(\lambda u+\mu v)\right)<0$ because otherwise $B\left(\left(f^{n_{k}}\right)^{\prime}(\lambda u+\mu v)\right)$ $>B\left(\left(f^{N}\right)^{\prime}(\lambda u+\mu v)\right) \geqq 0$, a contradiction with (1).

Then the subspaces spanned by $u, v$ would be in $S_{x} \Rightarrow S_{x}=T_{x} H$. This is absurd, and therefore $S_{x}, U_{x}$ are one-dimensional.

Since $f$ increases the values of $B, S_{x} \cap U_{x}=O_{x}$.

In order to prove the continuity of $S_{x}$ let $u_{n} \in S, u_{n} \rightarrow u \in T_{x} H$. Continuity of $B$ and positivity of $P$ (same arguments to the ones made with $w_{\infty}$ ) imply that

$$
B\left(\left(f^{n}\right)^{\prime} u\right)<0 \Rightarrow u \in S_{x} .
$$

In the following lemma the condition on $\log \left\|f_{x}^{\prime}\right\|$ - which indeed is a restriction on the behavior of $f$ near $K$-is needed to apply the ergodic multiplicative theorem of Osedelets [O1] that assures the existence of Lyapunov's exponents and proper spaces $v$-a.e. $w$.

Lemma 2. If the assumptions of Lemma 1 are fulfilled and

$$
\log ^{+}\left\|\left(f^{ \pm}\right)^{\prime}\right\| \in L^{1}(H, v), \text { where } \log ^{+} s=\max \{0, \log s\},
$$

then, if $R$ is the Pesin region for $f, v(R)=1$ and the proper spaces of $f$ on $x$ are $U_{x}, S_{x}$.

Proof. Let $U_{x}, S_{x}$ be as in the previous lemma for every $x \in H$, and $D \subset H$ compact.

There exist $a>0$ such $P(u)>a B(u)$ for every $u \in U_{x}, x \in D$. Then $B\left(f^{\prime}(u)\right)=B(u)$ $+P(u)>(1+a) B(u)$ and if $f^{n_{k}}(x) \in D$ for an increasing sequence $\left\{n_{k}\right\}$, it results:

$$
\begin{aligned}
B\left(\left(f^{n_{k}+1}\right)^{\prime} u\right) & >(1+a) B\left(\left(f^{n_{k}}\right)^{\prime} u\right)>(1+a) B\left(\left(f^{n_{k-1}+1}\right)^{\prime} u\right) \\
& \left.>(1+a)^{2} B\left(f^{n_{k}-1}\right)^{\prime} u\right)>\ldots>(1+a)^{k} B(u) .
\end{aligned}
$$

So $B\left(\left(f^{n}\right)^{\prime} u\right)>(1+a)^{N_{n}} B(u)$, where

$$
N_{n}=\operatorname{card}\left\{j: f^{j}(x) \in D, 0 \leqq j \leqq n-1\right\} \leqq n .
$$


Since $B$ is bounded there exists $b>0$ such that $|B(w)|<b\|w\|^{2} \forall w \in T H$. Then $\left\|\left(f^{n}\right)^{\prime} u\right\|^{2} \geqq \frac{1}{b}(1+a)^{N_{n}} B(u) \forall n \geqq 0$ and,

$$
\liminf \frac{1}{n} \log \left\|\left(f^{n}\right)^{\prime} u\right\| \geqq \frac{1}{2} \log (1+a) \liminf \frac{N_{n}}{n} .
$$

Applying the Birkhoff-Khinchin theorem to $X_{D}$, the characteristic function of $D$, we get that $v$-a.e. $w$. there exists

$$
\widetilde{X}_{D}(x)=\lim _{n \rightarrow+\infty} \frac{1}{n} \sum_{j=0}^{n-1} X_{D}\left(f^{j}(x)\right)=\lim _{n \rightarrow+\infty} \frac{N_{n}(x)}{n},
$$

and since

$$
v(D)=\int_{H} X_{D} d v=\int_{H} \bar{X}_{D} d v, \quad \lim _{n \rightarrow+\infty} \frac{N_{n}(x)}{n}
$$

is positive in $\bar{D}$ with $v(\bar{D}) \geqq v(D)$.

Now, if $u=v_{1}+v_{2}, v_{i} \in E_{i}(x)$, being $E_{i}(x)$ the proper subspaces of $f$ in the regular point $x$, associated to the exponent $\lambda_{i}(x), \lambda_{1}(x)>\lambda_{2}(x)$,

$$
\lim _{n \rightarrow+\infty} \frac{1}{n} \log \left(f^{n}\right)^{\prime} u \leqq \lim _{n \rightarrow+\infty} \frac{1}{n} \log 2\left\|\left(f^{n}\right)^{\prime} v_{1}\right\|=\lambda_{1}(x) .
$$

The positivity of the largest Lyapunov exponent in $D$ is deduced from this inequality and (2).

$E_{2}(x) \subset S_{x}$ because if $w \in E_{2}(x)$

$$
\lim _{n \rightarrow+\infty} \frac{1}{n} \log \left\|\left(f^{n}\right)^{\prime} w\right\|<0 \Rightarrow \lim _{n \rightarrow+\infty} \log \left\|\left(f^{n}\right)^{\prime} w\right\|=-\infty,
$$

$\lim _{n \rightarrow+\infty}\left\|\left(f^{n}\right)^{\prime} w\right\|=0, B\left(\left(f^{n}\right)^{\prime} w\right) \leqq 0 \forall n \in \mathbb{Z}$. The last step results from the boundedness of $B\left(B\left(\left(f^{n}\right)^{\prime} w<b\left\|\left(f^{n}\right)^{\prime} w\right\|^{2} \Rightarrow B\left(\left(f^{n}\right)^{\prime} w \leqq 0\right)\right.\right.$ and the positivity of $P$. Idem for $E_{1}(x) \subset U_{x}$ working with $n \rightarrow-\infty$. So $E_{2}(x)=S_{x}, E_{1}(x)=U_{x}$.

Acknowledgements. To Jorge Lewowicz (IME-FI, Montevideo); Artur Oscar Lopes (UFRGS, Porto Alegre); Pos-Graduação em Matemática, UFRGS; CAPES, Brasil.

\section{References}

[A1] Anosov, D.V., Sinai, Ya.G.: Some smooth ergodic systems. Russ. Math. Surv. 22:5, 103-67 (1967)

[A2] Arnold, V.I., Avez, A.: Problémes ergodiques de la Mécánique Classique. Paris: GauthierVillars 1967

[B1] Birkhoff, George D.: Dynamical systems. A.M.S. Colloquium Publications. Providence, RI: Am. Math. Soc. 1966 (Original ed. 1927)

[B2] Bunimovic, L.A.: On billiards close to dispersing. Math. USSR. Sb. 23, No. 1, 45-67 (1974)

[B3] Bunimovich, L.A.: On the ergodic properties of nowhere dispersing billiards. Commun. Math. Phys. 65, 295-312 (1979)

[C1] Cornfeld, I.P., Fomin, S.V., Sinai, Ya.G.: Ergodic theory. Berlin, Heidelberg, New York: Springer 1982 (Original ed. 1980) 
[K 1] Katok, A., Strelcyn, J.-M. in collaboration with Ledrappier, F., Przytycki, F.: Smooth maps with singularities. Invariant Manifolds, Entropy and Billiards. Université ParisNord. Prépublications Mathematiques No. 56 (1985). In: Lecture Notes in Mathematics, Vol. 1222. Berlin, Heidelberg, New York: Springer 1986

[G1] Gallavotti, G.: Lectures on the billiards. In: Lecture Notes in Physics, Vol. 38. Berlin, Heidelberg, New York: Springer 1975. Dynamical systems, theory and applications. Battelle Seattle 1974 Rencontres

[L1] Lewowicz, J.: Lyapunov functions and topological stability. J. Differ. Equations 38, 192-209 (1980)

[L2] Lewowicz, J.: Persistence in expansive systems. Ergodic Theory Dyn. Syst. 3, 567-578 (1983)

[L3] Lewowicz, J., Lima de Sa, E.: Analytical models of pseudo-Anosov maps. Ergodic Theory Dyn. Syst. 6, 385-392 (1986)

[L4] Lewowicz, J.: Lyapunov functions and stability of geodesic flows. Lecture Notes in Mathematics, Vol. 1007. Geometric dynamics. Proceedings, Rio de Janeiro 1981

[M1] Mañé, R.: Introdução à teoria ergôdica. Rio de Janeiro: IMPA 1983

[O1] Oseledets, V.I.: A multiplicative ergodic theorem. Lyapunov Characteristic numbers for dynamical systems. Trans. Mosc. Math. Soc. 19, 197-231 (1968)

[P1] Pesin, Ya.B.: Characteristic Lyapunov exponents and smooth ergodic theory. Russ. Math. Surv. 32:4, 55-112 (1977)

[S1] Sinai, Ya.G.: Dynamical systems with elastic reflactions. Russ. Math. Surv. 25:1, 137-189 (1970)

[W 1] Wojtkowski, M.: Principles for the design of billiards with nonvanishing Lyapunov exponents. Commun. Math. Phys. 105, 391-414 (1986)

Communicated by J.-P. Eckmann

Received July 13, 1987; in revised form December 3, 1987 
